\title{
65. Circular-linear model of the force of dry friction
}

\author{
K. Ragulskis ${ }^{1}$, A. Bubulis ${ }^{2}$, R. Maskeliūnas ${ }^{3}$, P. Paškevičius ${ }^{4}$, L. Ragulskis ${ }^{5}$ \\ ${ }^{1}$ Kaunas University of Technology, K. Donelaičio Str. 73, LT-44249, Kaunas, Lithuania \\ ${ }^{2}$ Kaunas University of Technology, Mechatronics Institute, Studentu Str. 56, LT-51424, Kaunas, Lithuania \\ ${ }^{3}$ Vilnius Gediminas Technical University, Saulètekio Al. 11, LT-10223, Vilnius, Lithuania \\ ${ }^{4}$ Company "Vaivora", Palemono Str. 2a, LT-52191, Kaunas, Lithuania \\ ${ }^{5}$ Vytautas Magnus University, Vileikos Str. 8, LT-44404, Kaunas, Lithuania \\ ${ }^{1}$ Corresponding author \\ E-mail: ${ }^{1}$ kazimieras3@hotmail.com, ${ }^{2}$ algimantas.bubulis@ktu.lt, ${ }^{3}$ rimas.maskeliunas@vgtu.lt, \\ 4info@vaivorairko.lt, ${ }^{5}$ l.ragulskis@if.vdu.lt
}

Received 30 October 2018; received in revised form 5 December 2018; accepted 13 December 2018 DOI https://doi.org/10.21595/mme.2018.20503

Check for updates

Copyright (C) 2018 K. Ragulskis, et al. This is an open access article distributed under the Creative Commons Attribution License, which permits unrestricted use, distribution, and reproduction in any medium, provided the original work is properly cited.

\begin{abstract}
In the numerical methods taking into account the force of dry friction numerical approximation is used when performing calculations. Circular-linear approximation for the local transition region is recommended. Results of numerical calculations are presented. The advantages of using this proposed representation are seen from the obtained results presented in this paper.
\end{abstract}

Keywords: circular-linear approximation, force of dry friction, numerical results, vibrations, graphical representation.

\section{Introduction}

When numerical methods are applied for nonlinear problems various types of procedures are used for approximation of nonlinear behavior of the system. In engineering applications it is important to take into account the force of dry friction. Various approximate representations of this phenomenon may be used. In this paper circular-linear approximation for the local transition region is proposed and investigated numerically.

This paper continues the investigations presented in [1] and [2]. Basic problems taking into account the force of dry friction and their analysis are presented in [3].

Problems of surface cleaning are described in [4]. This dynamical process is also presented in [5]. Particles and their interactions with surfaces are analyzed in [6]. Particles interacting with larger particles are investigated in [7]. Adhesion of particles is presented in [8]. Experimental investigations of particles are described in [9]. The force of dry friction is essential in the numerical analysis of models of cleaning of surfaces.

\section{Circular-linear approximation of dry friction}

Further $u$ denotes displacement of the investigated vibrating system, $H$ represents the approximate force representing dry friction and dot over the variable denotes differentiation with respect to the time $t$.

Value of velocity at the beginning of the local transition interval between the constant values of the force is $\dot{u}_{1}$ and value of velocity at the end of the local transition interval between the constant values of the force is $\dot{u}_{2}$. The average value is:

$\dot{u}_{c}=\frac{\dot{u}_{1}+\dot{u}_{2}}{2}$.

Value of the force approximating dry friction at the beginning of the local transition interval is $H_{1}$ and value of the force approximating dry friction at the end of the local transition interval is $\mathrm{H}_{2}$. The average value is: 
$H_{c}=\frac{H_{1}+H_{2}}{2}$

Further $r$ denotes small radius of the circle.

Case 1. It is assumed that:

$H_{1}>H_{2}$.

The following lengths are calculated:

$s=\sqrt{\left(\dot{u}_{c}-\dot{u}_{1}\right)^{2}+\left(\left(H_{1}-r\right)-H_{c}\right)^{2}}$,

$t=\sqrt{s^{2}-r^{2}}$.

The following angles are calculated:

$\alpha=\arctan \frac{r}{t^{\prime}}$
$\beta=\arctan \frac{\left(H_{1}-r\right)-H_{c}}{\dot{u}_{c}-\dot{u}_{1}}$.

The following coordinates of points are calculated:

$\dot{u}_{3}=\dot{u}_{c}-t \cos (\alpha+\beta)$,

$H_{3}=H_{c}+t \sin (\alpha+\beta)$,

$\dot{u}_{4}=\dot{u}_{c}+t \cos (\alpha+\beta)$,

$H_{4}=H_{c}-t \sin (\alpha+\beta)$.

The circular-linear special function is defined as:

$C\left(\dot{u}_{1}, \dot{u}_{2}, H_{1}, H_{2}, \dot{u}\right)= \begin{cases}H_{1}-r+\sqrt{r^{2}-\left(\dot{u}-\dot{u}_{1}\right)^{2}}, & \dot{u}<\dot{u}_{3}, \\ H_{2}+r-\sqrt{r^{2}-\left(\dot{u}-\dot{u}_{2}\right)^{2}}, & \dot{u}>\dot{u}_{4}, \\ H_{3}+\frac{H_{4}-H_{3}}{\dot{u}_{4}-\dot{u}_{3}}\left(\dot{u}-\dot{u}_{3}\right), & \dot{u}_{3} \leq \dot{u} \leq \dot{u}_{4} .\end{cases}$

Case 2. It is assumed that:

$H_{1}<H_{2}$.

The following lengths are calculated:

$s=\sqrt{\left(\dot{u}_{c}-\dot{u}_{1}\right)^{2}+\left(H_{c}-\left(H_{1}+r\right)\right)^{2}}$,

$t=\sqrt{s^{2}-r^{2}}$.

The following angles are calculated:

$\alpha=\arctan \frac{r}{t^{\prime}}$
$\beta=\arctan \frac{H_{c}-\left(H_{1}+r\right)}{\dot{u}_{c}-\dot{u}_{1}}$. 
The following coordinates of points are calculated:

$\dot{u}_{3}=\dot{u}_{c}-t \cos (\alpha+\beta)$,

$H_{3}=H_{c}-t \sin (\alpha+\beta)$,

$\dot{u}_{4}=\dot{u}_{c}+t \cos (\alpha+\beta)$,

$H_{4}=H_{c}+t \sin (\alpha+\beta)$.

The circular-linear special function is defined as:

$C\left(\dot{u}_{1}, \dot{u}_{2}, H_{1}, H_{2}, \dot{u}\right)= \begin{cases}H_{1}+r-\sqrt{r^{2}-\left(\dot{u}-\dot{u}_{1}\right)^{2}}, & \dot{u}<\dot{u}_{3}, \\ H_{2}-r+\sqrt{r^{2}-\left(\dot{u}-\dot{u}_{2}\right)^{2}}, & \dot{u}>\dot{u}_{4}, \\ H_{3}+\frac{H_{4}-H_{3}}{\dot{u}_{4}-\dot{u}_{3}}\left(\dot{u}-\dot{u}_{3}\right), & \dot{u}_{3} \leq \dot{u} \leq \dot{u}_{4} .\end{cases}$

The force of dry friction is approximated in the following way:

$H= \begin{cases}C(-\Delta, \Delta,-h, h, \dot{u}), & |\dot{u}|<\Delta, \\ h, & |\dot{u}| \geq \Delta, \quad \dot{u}>0, \\ -h, & |\dot{u}| \geq \Delta, \quad \dot{u}<0,\end{cases}$

where $h$ is the coefficient of dry friction, $\Delta$ determines the width of the interval of transition between the values of the force of dry friction.

The following system is investigated:

$m \ddot{u}+c \dot{u}+k u=P \sin \omega t-H$,

where $m$ is the mass, $c$ is the coefficient of viscous friction, $k$ is the stiffness, $P$ is the amplitude of the exciting force, $\omega$ is the frequency of the exciting force.

In the performed investigation values of the parameters of the system are: $\omega=1, h=3.2$, $P=4, m=1, c=0.1, k=1, r=0.15$. Zero initial conditions were assumed. Steady state motion was investigated, and two periods were represented.

Results for $\Delta=0.8$ are shown in Fig. 1 .

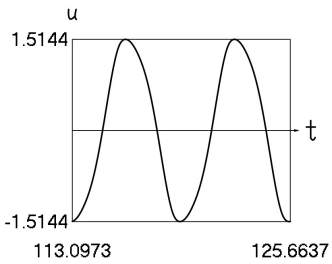

a) Displacement and time

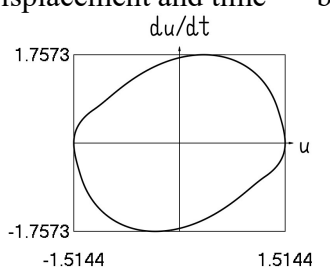

e) Velocity and displacement

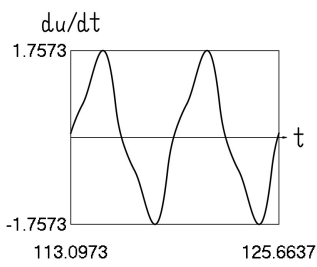

b) Velocity and time

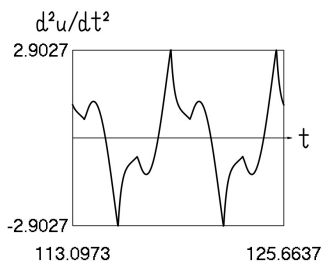

c) Acceleration and time

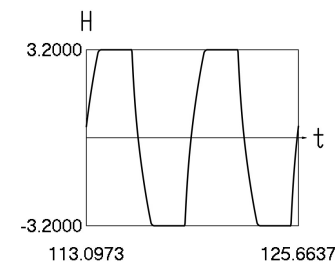

d) $H$ and time

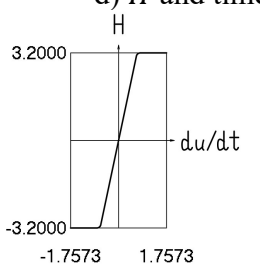

g) $H$ and velocity

Fig. 1. Dynamics in steady state regime (wide transition region)

Results for $\Delta=0.4$ are shown in Fig. 2 . 


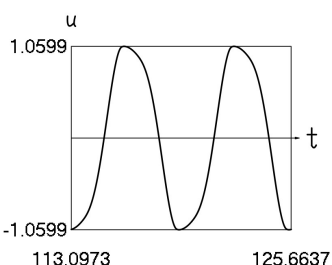

a) Displacement and time

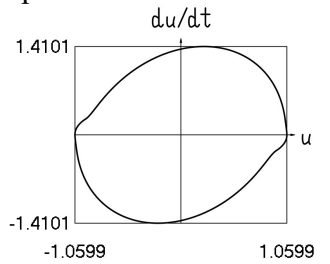

e) Velocity and displacement

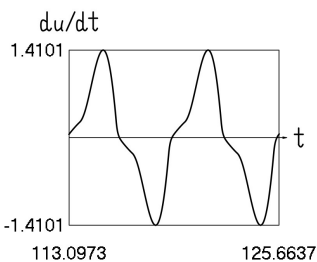

b) Velocity and time

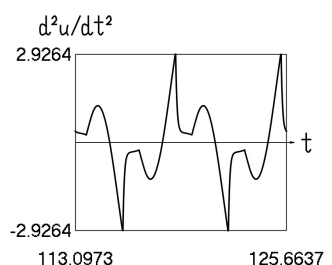

c) Acceleration and time

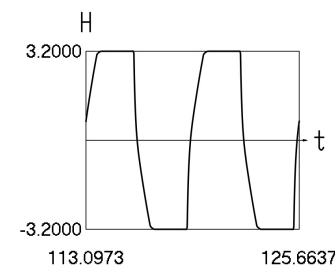

d) $H$ and time

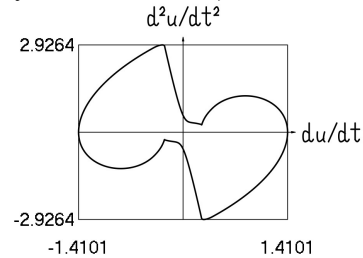

f) Acceleration and velocity

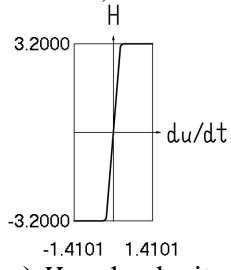

g) $H$ and velocity

Fig. 2. Dynamics in steady state regime (transition region of medium width)

Results for $\Delta=0.2$ are shown in Fig. 3 .

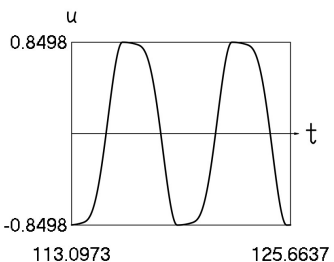

a) Displacement and time

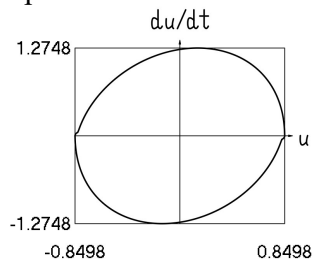

e) Velocity and displacement

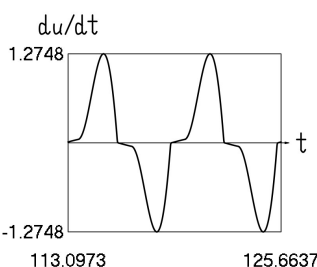

b) Velocity and time

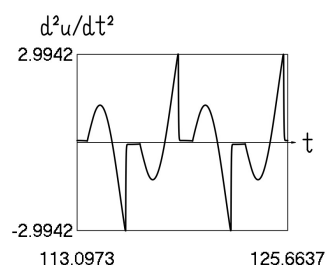

c) Acceleration and time

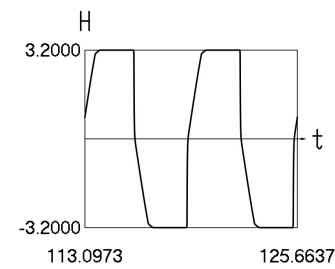

d) $H$ and time

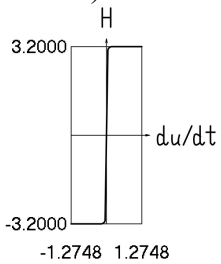

g) $H$ and velocity

Fig. 3. Dynamics in steady state regime (narrow transition region)

Three typical widths of the transition region are investigated. Typical results are presented. The effect of the width of the transition is seen from the obtained numerical results.

\section{More complicated model of dry friction}

The coefficient of dry friction increases in the vicinity of the value of zero velocity. It is assumed that $\frac{h}{\Delta}=\frac{h_{a}}{\Delta_{a}}$, where $h_{a}$ denotes the increase in the coefficient of dry friction, $\Delta_{a}$ defines the width of the transition interval connecting the values of the coefficient of dry friction $h$ and $h+h_{a}$. It follows that $h_{a}=h \frac{\Delta_{a}}{\Delta}$.

The force of dry friction is approximated in the following way: 


$$
H= \begin{cases}C\left(-\Delta-\Delta_{a}, \Delta+\Delta_{a},-h-h \frac{\Delta_{a}}{\Delta}, h+h \frac{\Delta_{a}}{\Delta}, \dot{u}\right), & |\dot{u}|<\Delta+\Delta_{a}, \\ C\left(\Delta+\Delta_{a}, \Delta+2 \Delta_{a}, h+h \frac{\Delta_{a}}{\Delta}, h, \dot{u}\right), & \Delta+\Delta_{a} \leq|\dot{u}|<\Delta+2 \Delta_{a}, \dot{u}>0, \\ C\left(-\Delta-2 \Delta_{a},-\Delta-\Delta_{a},-h,-h-h \frac{\Delta_{a}}{\Delta}, \dot{u}\right), & \Delta+\Delta_{a} \leq|\dot{u}|<\Delta+2 \Delta_{a}, \dot{u}<0, \\ h, & |\dot{u}| \geq \Delta+2 \Delta_{a}, \dot{u}>0, \\ -h, & |\dot{u}| \geq \Delta+2 \Delta_{a}, \dot{u}<0 .\end{cases}
$$

In the performed investigation values of the parameters of the system are: $\omega=1, h=1.6$, $\Delta=1.8, \Delta_{a}=1.8, P=4, m=1, c=0.1, k=1, r=0.8$. Zero initial conditions were assumed. Steady state motion was investigated and two periods were represented.

Results are shown in Fig. 4.

Dynamics of the system with more complicated model of dry friction is seen from the obtained numerical results.

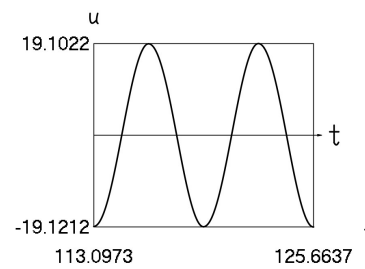

a) Displacement and time

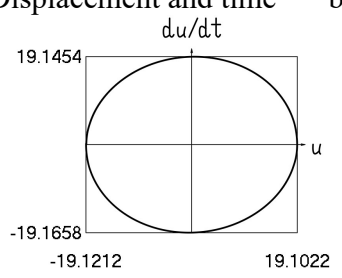

e) Velocity and displacement

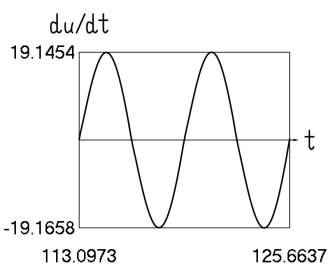

b) Velocity and time

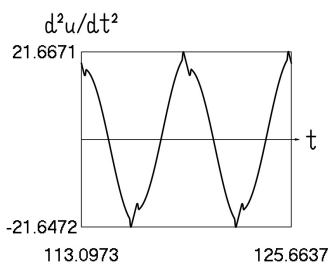

c) Acceleration and time

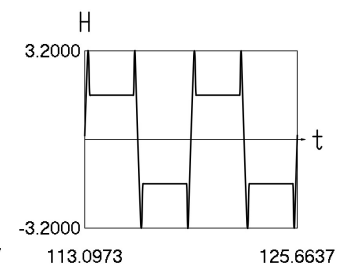

d) $H$ and time

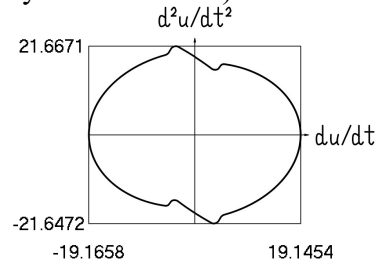

f) Acceleration and velocity

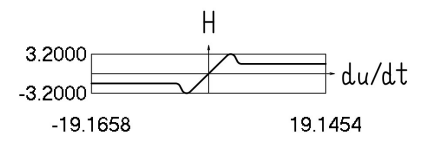

g) $H$ and velocity

Fig. 4. Dynamics in steady state regime (more complicated model of dry friction)

\section{Conclusions}

When performing numerical analysis of engineering problems in which the force of dry friction is essential for the representation of behavior of the dynamical system some type of numerical approximation is used in the process of calculations. In this paper circular-linear approximation for the local transition region is investigated. The advantages of using this proposed representation are seen from the presented graphical results.

Three typical widths of the transition region are investigated. Typical results are presented. The effect of the width of the transition is seen from the obtained numerical results.

In the more complicated model of dry friction the coefficient of dry friction increases in the vicinity of the value of zero velocity. Dynamics of the system with more complicated model of dry friction is seen from the graphically presented numerical results.

On the basis of the obtained numerical results circular-linear approximation of dry friction is recommended for calculations in the investigations of engineering problems. Among those problems the investigations of processes of surface cleaning are essential.

\section{Acknowledgements}

The authors thank the reviewers for providing ideas and valuable comments. They enabled to 
improve the presentation in the paper.

\section{References}

[1] Ragulskis K., Bubulis A., Paškevičius P., Pauliukas A., Ragulskis L. Investigation of elliptical approximation in the model of the force of dry friction. Mathematical Models in Engineering, Vol. 4, Issue 3, 2018, p. 151-156.

[2] Ragulskis K., Paškevičius P., Bubulis A., Pauliukas A., Ragulskis L. Improved numerical approximation of dry friction phenomena. Mathematical Models in Engineering, Vol. 3, Issue 2, 2017, p. 106-111.

[3] Sumbatov A. S., Yunin Ye. K. Selected Problems of Mechanics of Systems with Dry Friction. Physmathlit, Moscow, 2013, (in Russian).

[4] Chahine G. L., Kapahi A., Choi J.-K., Hsiao Ch.-T. Modeling of surface cleaning by cavitation bubble dynamics and collapse. Ultrasonics Sonochemistry, Vol. 29, 2016, p. 528-549.

[5] Witte A. K., Bobal M., David R., Blättler B., Schoder D., Rossmanith P. Investigation of the potential of dry ice blasting for cleaning and disinfection in the food production environment. LWT Food Science and Technology, Vol. 75, 2016, p. 735-741.

[6] Petean P. G. C., Aguiar M. L. Determination of the adhesion force between particles and rough surfaces. Powder Technology, Vol. 274, 2015, p. 67-76.

[7] Cui Y., Sommerfield M. Forces on micron-sized particles randomly distributed on the surface of larger particles and possibility of detachment. International Journal of Multiphase Flow, Vol. 72, 2015, p. 39-52.

[8] Jiang Y., Turner K. T. Measurement of the strength and range of adhesion using atomic force microscopy. Extreme Mechanics Letters, Vol. 9, Issue 1, 2016, p. 119-126.

[9] Kumar N., Zhao C., Klaassen A., Van Den Ende D., Mugele F., Siretanu I. Characterisation of the surface charge distribution on kaolinite particles using high resolution atomic force microscopy. Geochimica et Cosmochimica Acta, Vol. 175, 2016, p. 100-112. 